\title{
COMPOSICIÓN Y ESTRUCTURA DE LA COMUNIDAD MACROBENTÓNICA EN EL SISTEMA INTERIOR DE CANALES Y FIORDOS DEL EXTREMO AUSTRAL DE CHILE
}

\author{
MACROBENTHIC COMPOSITION AND STRUCTURE IN THE INNER CHILEAN \\ SOUTHERN CHANNEL AND FJORDS SYSTEM
}

Carlos Ríos $^{1}$, Erika Mutschke ${ }^{1} \&$ Américo Montiel ${ }^{1}$

\begin{abstract}
The composition of the community and the distribution and abundance of benthic macroinvertebrates, was investigated in the inland channels and fiords of Southern Chile. The field work was carried out during the 15 CIMAR FIORDS Cruise, on the Chilean Navy oceanographic vessel "AGOR Vidal Gormaz". A modified trawl net Agassiz (AGT) was used to record semi-quantitative data from 19 sampling stations, with depths between 26-620 m. A total of 61 taxa were identified from 13 stations with successful samples. Molluscs (16 taxa) and echinoderms (15 taxa) were the dominant groups in terms of species richness, while echinoderms were numerically the largest group (54\%). The asteroid Ctenodiscus procurator was the dominant species $(43 \%)$ in all stations. The results show spatial differences in structure and composition of macro- invertebrates, suggesting the existence of two species assemblages associated with depth as the main factor. It is suggested that the biodiversity in these areas remains relatively high due to a high turnover of species between sites, with well-defined sectors and also differentiation of its biotic composition. Future studies would be improved by a greater density of sampling stations and the inclusion of quantitative methods of sampling complementing the semiquantitative with an AGT.
\end{abstract}

Key word: benthos, marine communities, biodiversity, species richness, Magellan, subantarctic, CIMAR, EstimateS. 


\section{RESUMEN}

La composición de la comunidad, en conjunto con la distribución y abundancia de los macroinvertebrados bentónicos, fue estudiada en el sector de canales y fiordos interiores de la zona austral de Chile. El trabajo de terreno fue realizado durante el Crucero CIMAR 15 FIORDOS, a bordo del buque oceanográfico de la Armada de Chile "AGOR Vidal Gormaz". Se utilizó una red de arrastre Agassiz modificada (AGT) para tomar datos semi-cuantitativos en 19 estaciones de muestreo, con profundidades que variaron entre 26-620 m. Un total de 61 taxones fueron identificados en 13 estaciones con lances exitosos. Los moluscos (16 taxones) y los equinodermos (15 taxones) fueron los grupos dominantes en términos de riqueza específica, en tanto que numéricamente los equinodermos fueron el grupo mayoritario (54\%). El asteroideo Ctenodiscus procurator resultó ser la especie dominante (43\%) en el conjunto de estaciones. Los resultados mostraron diferencias espaciales en la estructura y composición de macroinvertebrados, sugiriendo la existencia de dos ensambles asociados a la profundidad como factor principal. Se sugiere que la relativamente alta biodiversidad en estas áreas se mantiene por un alto remplazo de especies entre sitios, con sectores bien definidos y además diferenciados respecto de su composición biótica. Para futuros estudios, es altamente deseable una mayor densidad de estaciones de muestreo y la inclusión de métodos cuantitativos de toma de muestras complementarios a los semicuantitativos con una AGT.

Palabras clave: bentos, comunidades marinas, biodiversidad, riqueza de especies, Magallanes, subantártica, CIMAR, EstimateS.

\section{INTRODUCCIÓN}

La zona de los canales y fiordos australes chilenos es una de las extensiones estuarinas más vastas y complejas del planeta (Silva \& Palma, 2008). Su extensión geográfica, ca. 84.000 $\mathrm{km}$ de costa lineal (Mutschke, 2008) y su origen asociado a la historia glaciar pasada y su influencia actual en el cono sur de Sudamérica (Clapperton et al. 1995; McCulloch et al. 1997; Kilian et al. 2007), generan una enorme variedad de hábitats específicos ecológicamente jóvenes (Camus, 2001) y de numerosos tipos de comunidades asociadas (cf. Arntz et al. 2005). Consecuentemente, la diversidad se presenta con una amplia heterogeneidad de valores. Como prueba de ello, se ha descrito que la riqueza de especies que componen las comunidades bentónicas presentes en los fondos blandos de la región de canales y fiordos varia entre 131 especies (Ríos et al. 2003; 2007; 2010a; Mutschke, 2008) hasta 1.400 (Ríos, 2007).

Sin embargo, varios aspectos deficitarios que se reconocen globalmente como negativos para un adecuado conocimiento de la biodiversidad marina, tales como un muestreo disperso, no uniforme y no representativo (e.g. Costello et al. 2010) son claramente aplicables para el caso de los ambientes marinos del extremo sur de Sudamérica. En esta zona, la mayoría de los hábitats marinos no han sido prospectados $e$ investigados $\mathrm{y}$, adicionalmente, muchos grupos taxonómicos bien diversificados y pequeños permanecen hasta la fecha escasa o nulamente estudiados. Esta situación contrasta negativamente con la importancia económica y ecológica que se le otorga al área marina austral. Por una lado, se la señala como sector relevante para contribuir a la explotación y el cultivo de recursos marinos y, por otro, se le considera clave para contribuir a explicar patrones ecológicos y biogeográficos a lo largo de la costa del Pacífico o gradientes de diversidad hacia o desde los ecosistemas marinos antárticos (Brattström \& Johanssen, 1983; Lancellotti \& Vásquez, 2000; Gray, 2001; Arntz \& Ríos, 1999; Camus, 2001; Arntz et al. 2005; Fernández et al. 2000; Escribano et al. 2003; Valdovinos et al. 2003; Montiel et al. 2005). 

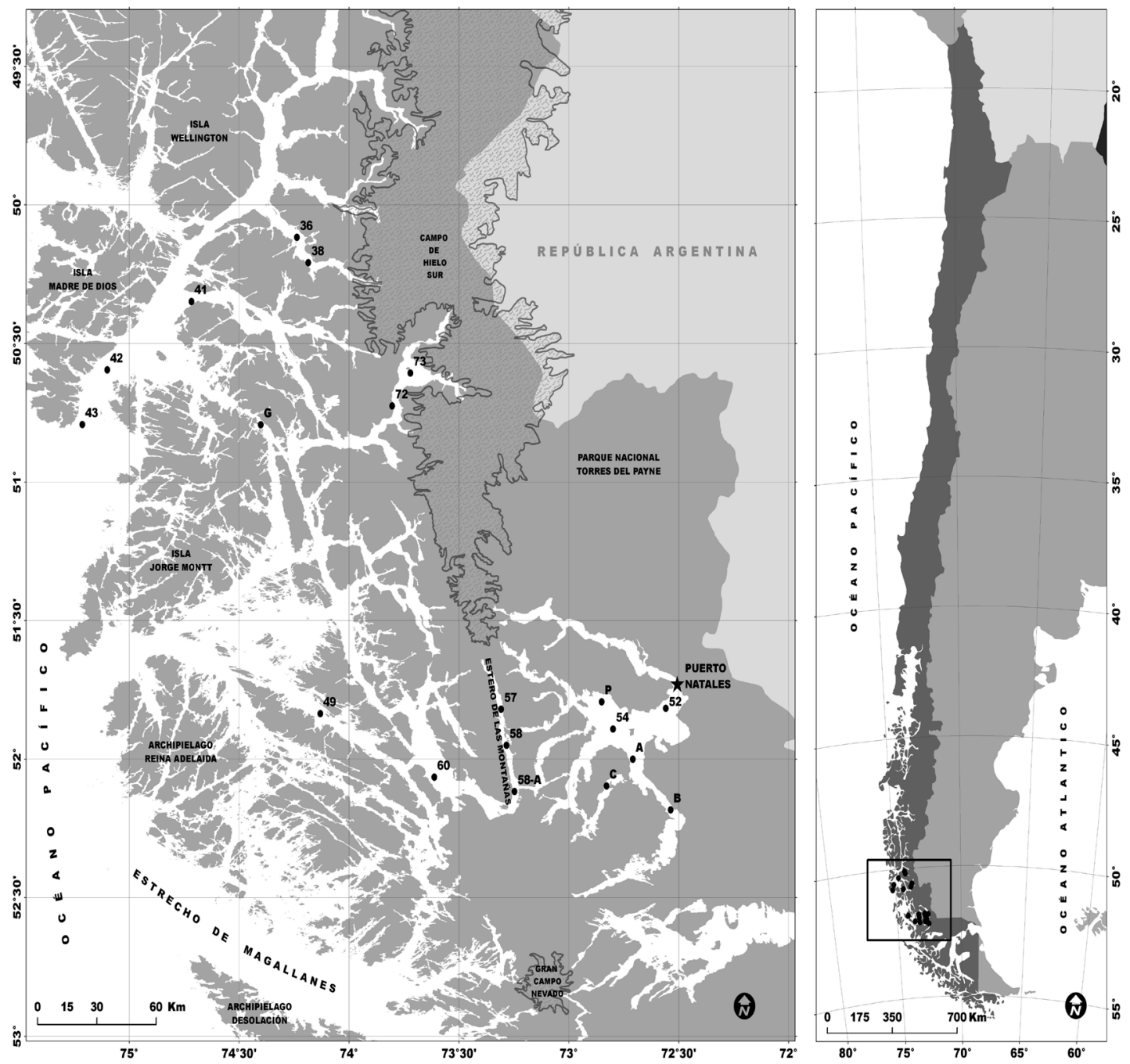

Fig. 1. Localización de las estaciones de muestreo contempladas para la caracterización de la macrofauna epibentónica durante el Crucero CIMAR 15 FIORDOS.

Durante el año 2009, el Comité Oceanográfico Nacional llevo a cabo el decimo quinto crucero de investigación científico-marina en el borde más septentrional de Magallanes. Este crucero tuvo como objetivo general contribuir a la obtención de información básica sobre la biodiversidad y oceanografía, generando así herramientas predictivas frente los posibles cambios en el ecosistema marino frente a alteraciones de tipo natural y/o antropogénica de una de las áreas menos prospectada de la región (CONA 2010¹).

En este contexto, el presente estudio analiza la composición de especies macrobentónicas, en conjunto con sus abundancias y distribuciones, a fin de caracterizar estructuralmente los ensambles presentes entre el seno Europa y el seno Obstrucción, localizados en el complejo de fiordos y canales del cono sur de Sudamérica.

1 CONA. 2010. Introducción. En: Crucero CIMAR 15 Fiordos: Informes preliminares. pp. 11-12. Comité Oceanográfico Nacional, Chile. 
Tabla 1. Antecedentes de las estaciones de muestreo de macrofauna epibentónica durante el crucero CIMAR 15 FIORDOS, utilizando una red de arrastre Agassiz modificada.

\begin{tabular}{|c|c|c|c|c|c|c|c|c|}
\hline ESTACIONES & Localidad & $\begin{array}{c}\text { Fecha de } \\
\text { muestreo } \\
2009\end{array}$ & Latitud & Longitud & $\begin{array}{l}\text { Profundidad } \\
\text { promedio } \\
\text { (m) }\end{array}$ & $\begin{array}{c}\text { Tiempo } \\
\text { arrastre } \\
\text { (min) }\end{array}$ & $\mathrm{N}^{\circ}$ Ind & $\begin{array}{l}\mathrm{N}^{\circ} \\
\text { Esp. }\end{array}$ \\
\hline VG09-36 & Seno Europa & $18-10$ & $50^{\circ} 07,0^{\prime \prime}$ & $74^{\circ} 14,2^{\prime \prime}$ & 250 & 15 & 40 & 5 \\
\hline VG09-38 & Seno Europa & $18-10$ & $50^{\circ} 12,5^{\prime \prime}$ & $74^{\circ} 11,1^{\prime \prime}$ & 414 & 15 & 0 & 0 \\
\hline VG09-41 & Canal Concepción & $19-10$ & $50^{\circ} 20,9^{\prime \prime}$ & $74^{\circ} 43,0^{\prime \prime}$ & 410 & 15 & 46 & 4 \\
\hline VG09-42 & Canal Concepción & $19-10$ & $50^{\circ} 35,7 ”$ & $75^{\circ} 06,0^{\prime \prime}$ & 517 & 15 & 0 & 0 \\
\hline VG09-73 & Estero Andrés & $21-10$ & $50^{\circ} 36,40^{\prime \prime}$ & $73^{\circ} 43,3 "$ & 75 & 15 & 0 & 0 \\
\hline VG09-57 & Canal de las Montañas & $06-11$ & $51^{\circ} 49,0^{\prime \prime}$ & $73^{\circ} 14,4 "$ & 125 & 15 & 353 & 23 \\
\hline VG09-58A & Canal de las Montañas & $07-11$ & $52^{\circ} 07,0^{\prime \prime}$ & $73^{\circ} 14,8^{\prime \prime}$ & 280 & 15 & 968 & 24 \\
\hline VG09-54 & Golfo Almte. Montt & 03-11 & $51^{\circ} 53,5^{\prime \prime}$ & $72^{\circ} 47,9 "$ & 83 & 15 & 0 & 0 \\
\hline VG09-A & Seno Obstrucción & 04-11 & $52^{\circ} 00,0^{\prime \prime}$ & $72^{\circ} 42,5^{\prime \prime}$ & 45 & 15 & 0 & 0 \\
\hline VG09-B & Seno Obstrucción & 04-11 & $52^{\circ} 11,0^{\prime \prime}$ & $72^{\circ} 32,2^{\prime \prime}$ & 26 & 15 & 1 & 2 \\
\hline VG09-C & Seno Poca Esperanza & 01-11 & $52^{\circ} 05,8^{\prime \prime}$ & $72^{\circ} 49,7$ & 200 & 15 & 0 & 0 \\
\hline VG09-72 & Estero Peel & $21-10$ & $50^{\circ} 43,5^{\prime \prime}$ & $73^{\circ} 48,2^{\prime \prime}$ & 354 & 15 & 21 & 9 \\
\hline VG09-G & Angostura Guía & $23-10$ & $50^{\circ} 47,6 ”$ & $74^{\circ} 24,1^{\prime \prime}$ & 304 & 15 & 79 & 8 \\
\hline VG09-43 & Canal Concepción & $25-10$ & $50^{\circ} 47,6 ”$ & $75^{\circ} 12,8^{\prime \prime}$ & 254 & 10 & 6 & 8 \\
\hline VG09-52 & Seno Última Esperanza & 04-11 & $51^{\circ} 49,0^{\prime \prime}$ & $72^{\circ} 33,5^{\prime \prime}$ & 25 & 15 & 5 & 3 \\
\hline VG09-P & Golfo Almte. Montt & 03-11 & $51^{\circ} 47,6^{\prime \prime}$ & $72^{\circ} 51,0 ”$ & 170 & 15 & 29 & 4 \\
\hline VG09-58 & Canal de las Montañas & 06-11 & $51^{\circ} 57,0^{\prime \prime}$ & $73^{\circ} 17,0^{\prime \prime}$ & 140 & 15 & 1343 & 23 \\
\hline VG09-60 & Seno Unión & 07-11 & $52^{\circ} 03,9 ”$ & $73^{\circ} 36,7^{\prime \prime}$ & 170 & 15 & 91 & 18 \\
\hline VG09-49 & Canal Smyth & $09-11$ & $51^{\circ} 50,2^{\prime \prime}$ & $74^{\circ} 07,8^{\prime \prime}$ & 620 & 15 & 139 & 10 \\
\hline
\end{tabular}

\section{MATERIALES Y MÉTODOS}

Área de estudio

El área de estudio está localizada entre el seno Europa (5007,00"; $\left.74^{\circ} 14,20^{\prime \prime}\right)$ y el seno Obstrucción (52¹1,00"; 72³2,20"; Fig. 1), en la porción sur de la Zona Central de Canales y Fiordos Chilenos (sensu Silva \& Palma, 2008). El sector se caracteriza por ser una zona de aguas interiores, con mezclamientos de aguas de origen subantártico con aguas no salinas en distintas proporciones, conforme al aporte de ríos, ventisqueros, escurrimiento costero y pluviosidad (Sievers \& Silva, 2006). Los sitios más expuestos a la influencia del Océano Pacífico considerados en este estudio (e.g. canal Concepción, angostura Guía, canal Smyth) presentan contenidos de carbono y de nitrógeno orgánico relativamente altos en comparación con valores determinados para sitios más interiores (e.g. seno Obstrucción, golfo Almirante Montt, estero Peel, seno Europa). De acuerdo a los datos de Silva (2010), durante el período de estudio las temperaturas superficiales del agua de mar varían entre 6,0 y $7,2^{\circ} \mathrm{C}$, con una profundización de las isotermas hacia el interior del golfo Almirante Montt, mientras que la salinidad superficial fluctúa entre 14,6 en sitios más interiores (e.g. canal Señoret) y 29,3 psu en sitios más asociados a la influencia del Océano Pacífico (e.g. canal Smyth). 


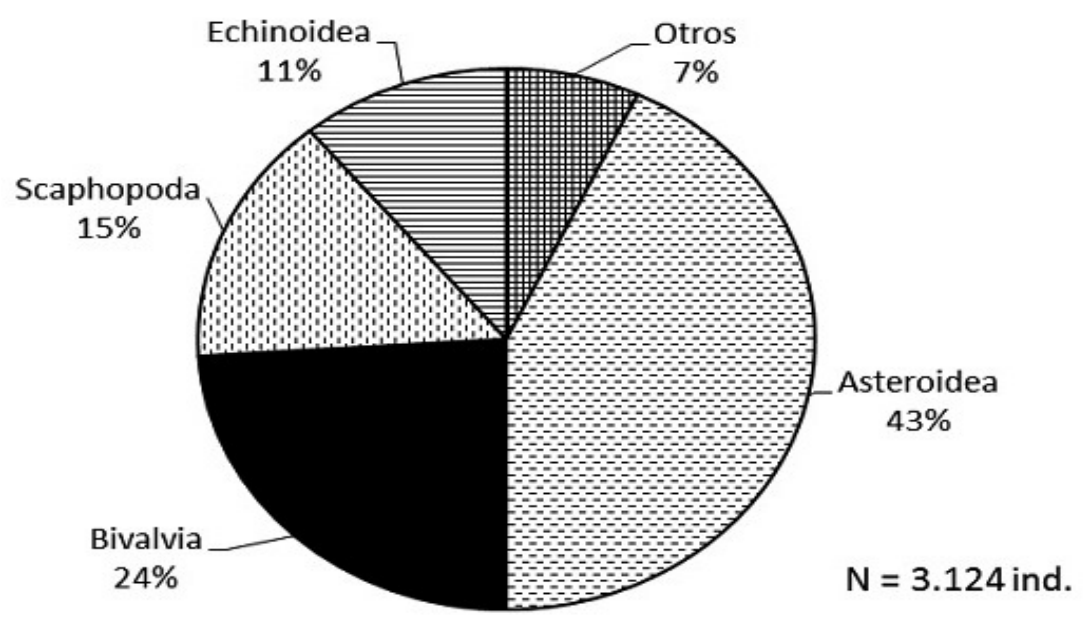

Fig. 2. Representación porcentual de cada taxa bentónico, basado en datos de número de individuos, obtenidos durante el Crucero CIMAR 15 FIORDOS.

Trabajo en terreno y determinación del material

Los especímenes recolectados fueron obtenido durante el Crucero CIMAR 15 FIORDOS, realizado a bordo del buque oceanográfico de la Armada de Chile "AGOR Vidal Gormaz" (Ríos et al. 2010b). Las muestras de sedimentos fueron recolectadas en 19 sitios (Fig. 1) utilizando una rastra Agassiz (AGT) modificada (3,15 $\mathrm{m}$ de ancho y 1,1 m de alto, con un tamaño de red de $10 \mathrm{~mm}$ ). El promedio de tiempo de arrastre en el fondo fue de 14,7 minutos y el rango de profundidad vario entre 26 - $620 \mathrm{~m}$ (Tabla 1).

La totalidad del sedimento recolectado en cada sitio por la AGT fue cernido in-situ utilizando tamices con una abertura de malla de 0,5 mm. Todos los organismos retenidos fueron preservados en alcohol al $80 \%$ y transportados al Laboratorio de Hidrobiología (Instituto de la Patagonia, Universidad de Magallanes) para su posterior determinación al nivel taxonómico más bajo posible.

De los 19 sitios en donde se realizaron lances con AGT, en seis no hubo evidencias de especímenes bentónicos. De esta manera, todos los análisis posteriores están basados en un total de 13 estaciones (Tabla 1 ).
Para la identificación del material recolectado se emplearon los trabajos de Bernasconi (1969) y Bernasconi \& D'Agostino (1971, 1977) para equinodermos; Castellanos (1988, 1992, 1994), Forcelli (2000), Osorio \& Reid (2004) y Cárdenas et al. (2008) para moluscos; Hartmann-Schröder (1965), Rozbaczylo (1980) para poliquetos, Menzies (1962), Retamal (1974) para crustáceos y Lamilla \& Bustamante (2005) para peces. Además, la identificación taxonómica fue complementada con aportes de especialistas en grupos específicos. El material fue depositado en la Sala de Colecciones "Edmundo Pisano V." del Instituto de la Patagonia, Universidad de Magallanes.

Análisis de los datos

Para estimar la biodiversidad del área de estudio se utilizó el estimado CHAO1, el cual sólo emplea el número de individuos por especie. Para este análisis se definió la muestra como "la especie por lance" (Colwell, 2006). El estimador CHAO1 fue calculado mediante el programa EstimateS (EstimateS 8.2; http://viceroy.eeb.uconn.edu/ estimates).

Basado en el número de individuos, 
Tabla 2. Número de individuos por taxa recolectado con una red Agassiz modificada durante el Crucero CIMAR 15 FIORDOS.

\begin{tabular}{|c|c|c|c|c|c|c|c|c|c|c|c|c|c|c|}
\hline TAXA\ESTACIÓN & $\begin{array}{l}\text { VGO } \\
36\end{array}$ & $\begin{array}{l}\text { VGO } \\
41\end{array}$ & $\begin{array}{l}\text { VGO } \\
57\end{array}$ & $\begin{array}{l}\text { VGO } \\
58 \mathrm{~A}\end{array}$ & $\underset{\mathrm{B}}{\mathrm{VGO}}$ & $\begin{array}{l}\text { VGO } \\
72\end{array}$ & $\underset{G}{\text { VGO }}$ & $\underset{43}{\mathrm{VGO}}$ & $\begin{array}{l}\text { VGO } \\
52\end{array}$ & $\underset{\mathrm{P}}{\mathrm{VGO}}$ & $\begin{array}{l}\text { VGO } \\
58\end{array}$ & $\begin{array}{l}\text { VGO } \\
60\end{array}$ & $\begin{array}{l}\text { VGO } \\
49\end{array}$ & TOTAL \\
\hline Porifera Indet. & 0 & 0 & 0 & 0 & 0 & 0 & 0 & 0 & 0 & 0 & 0 & 1 & 1 & 2 \\
\hline Hidrozoa Indet. & 0 & 0 & 1 & 0 & 0 & 0 & 0 & 1 & 0 & 0 & 1 & 0 & 0 & 3 \\
\hline Aphroditidae Indet. & 0 & 0 & 0 & 0 & 0 & 1 & 0 & 0 & 0 & 0 & 0 & 0 & 0 & 1 \\
\hline Pareurythoe chilensis & 0 & 0 & 0 & 0 & 0 & 0 & 0 & 1 & 0 & 0 & 0 & 0 & 0 & 1 \\
\hline Onuphis pseudoiridescens & 0 & 0 & 0 & 0 & 0 & 2 & 0 & 0 & 0 & 0 & 0 & 0 & 1 & 3 \\
\hline Leanira quatrefagesi & 0 & 0 & 1 & 0 & 0 & 1 & 0 & 0 & 1 & 0 & 4 & 1 & 0 & 8 \\
\hline Abyssoninoe sp. & 0 & 0 & 0 & 0 & 0 & 0 & 0 & 0 & 1 & 0 & 0 & 0 & 0 & 1 \\
\hline Ninoe cf. leptognatha & 0 & 0 & 2 & 0 & 0 & 0 & 0 & 0 & 0 & 0 & 0 & 0 & 0 & 2 \\
\hline Lumbrineris cingulata & 0 & 0 & 2 & 0 & 0 & 0 & 1 & 0 & 0 & 0 & 0 & 0 & 0 & 3 \\
\hline Abyssoninoe abyssorum & 0 & 0 & 0 & 0 & 0 & 0 & 0 & 0 & 0 & 0 & 0 & 1 & 0 & 1 \\
\hline Terebellides stroemi & 0 & 0 & 1 & 0 & 0 & 0 & 0 & 0 & 0 & 0 & 0 & 0 & 0 & 1 \\
\hline Aglaophamus virgini & 0 & 0 & 0 & 0 & 0 & 0 & 0 & 0 & 0 & 0 & 0 & 1 & 0 & 1 \\
\hline Notomastus latericeus & 0 & 0 & 0 & 0 & 0 & 0 & 0 & 0 & 0 & 0 & 0 & 1 & 0 & 1 \\
\hline Drilonereis tenuis & 0 & 0 & 0 & 0 & 0 & 2 & 0 & 0 & 0 & 0 & 0 & 0 & 0 & 2 \\
\hline Tindaria sp. & 0 & 0 & 1 & 0 & 0 & 0 & 0 & 0 & 0 & 0 & 0 & 0 & 0 & 1 \\
\hline Aulacomya ater & 0 & 0 & 1 & 8 & 0 & 0 & 0 & 0 & 0 & 0 & 0 & 0 & 0 & 9 \\
\hline Limopsis marionensis & 0 & 0 & 2 & 1 & 0 & 0 & 0 & 0 & 0 & 0 & 1 & 2 & 0 & 6 \\
\hline Eunnucula grayi & 0 & 0 & 3 & 0 & 0 & 0 & 0 & 0 & 0 & 0 & 0 & 0 & 0 & 3 \\
\hline Nuculoidea Indet. & 0 & 0 & 14 & 41 & 0 & 0 & 28 & 0 & 0 & 0 & 174 & 0 & 0 & 257 \\
\hline Propeleda longicaudata & 0 & 0 & 0 & 0 & 0 & 0 & 0 & 0 & 0 & 0 & 0 & 1 & 0 & 1 \\
\hline Pandora cistula & 0 & 0 & 0 & 12 & 0 & 0 & 0 & 0 & 0 & 0 & 70 & 0 & 0 & 82 \\
\hline Cyclocardia velutinus & 0 & 0 & 0 & 9 & 0 & 0 & 0 & 0 & 0 & 0 & 2 & 2 & 0 & 13 \\
\hline Carditidae Indet. & 0 & 0 & 6 & 8 & 0 & 0 & 0 & 0 & 0 & 0 & 322 & 0 & 0 & 336 \\
\hline Lucinoma antartica & 0 & 0 & 0 & 0 & 0 & 0 & 0 & 0 & 0 & 22 & 0 & 0 & 0 & 22 \\
\hline Darina selenoides & 0 & 0 & 0 & 0 & 0 & 0 & 0 & 0 & 0 & 0 & 0 & 0 & 3 & 3 \\
\hline Tellina petittiana & 0 & 0 & 0 & 0 & 0 & 0 & 0 & 0 & 0 & 0 & 1 & 0 & 0 & 1 \\
\hline Pectinidae Indet. & 0 & 0 & 0 & 10 & 0 & 0 & 0 & 0 & 0 & 0 & 0 & 0 & 0 & 10 \\
\hline Acesta patagonica & 0 & 0 & 0 & 1 & 0 & 0 & 0 & 0 & 0 & 0 & 0 & 0 & 0 & 1 \\
\hline $\begin{array}{l}\text { Siphonodentalium } \\
\text { falklandicum }\end{array}$ & 0 & 0 & 0 & 2 & 0 & 3 & 2 & 0 & 0 & 0 & 7 & 0 & 1 & 15 \\
\hline Rhabdus perceptum & 0 & 2 & 0 & 1 & 0 & 0 & 20 & 0 & 0 & 0 & 3 & 0 & 50 & 76 \\
\hline Dentalium (F.) majorinum & 1 & 0 & 0 & 221 & 0 & 4 & 25 & 0 & 0 & 0 & 108 & 23 & 0 & 382 \\
\hline Magellania venosa & 0 & 0 & 0 & 0 & 0 & 2 & 0 & 0 & 0 & 0 & 0 & 0 & 0 & 2 \\
\hline Peltarion spinosulum & 0 & 0 & 2 & 1 & 2 & 0 & 0 & 0 & 3 & 0 & 0 & 0 & 0 & 8 \\
\hline Acanthocyclus cf. albatrossis & 0 & 0 & 0 & 0 & 0 & 0 & 0 & 0 & 0 & 0 & 2 & 0 & 0 & 2 \\
\hline Libidoclea smithi & 0 & 0 & 4 & 4 & 0 & 0 & 0 & 0 & 0 & 0 & 13 & 0 & 0 & 21 \\
\hline Edotea sp. & 0 & 0 & 0 & 1 & 0 & 0 & 1 & 0 & 0 & 0 & 0 & 0 & 0 & 2 \\
\hline Pasiphaea acutifrons & 0 & 0 & 8 & 0 & 0 & 0 & 0 & 0 & 0 & 0 & 0 & 0 & 0 & 8 \\
\hline Austropandalus grayi & 0 & 0 & 0 & 12 & 0 & 0 & 0 & 0 & 0 & 1 & 16 & 0 & 0 & 29 \\
\hline Munidopsis opalescens & 0 & 0 & 2 & 0 & 0 & 0 & 0 & 0 & 0 & 0 & 0 & 0 & 0 & 2 \\
\hline Munida subrugosa & 0 & 0 & 0 & 1 & 0 & 0 & 1 & 0 & 0 & 0 & 1 & 0 & 2 & 5 \\
\hline Porianopsis echinaster & 0 & 0 & 0 & 1 & 0 & 0 & 0 & 0 & 0 & 0 & 0 & 0 & 0 & 1 \\
\hline Bathybiaster loripes & 0 & 7 & 0 & 0 & 0 & 0 & 0 & 0 & 0 & 0 & 0 & 0 & 6 & 13 \\
\hline Odontaster penicillatus & 0 & 0 & 0 & 0 & 0 & 0 & 0 & 0 & 0 & 3 & 0 & 1 & 0 & 4 \\
\hline Anasterias antarctica & 0 & 0 & 8 & 0 & 0 & 0 & 0 & 2 & 0 & 3 & 0 & 2 & 0 & 15 \\
\hline Cosmasterias lurida & 0 & 0 & 0 & 0 & 0 & 0 & 0 & 1 & 0 & 0 & 0 & 2 & 1 & 4 \\
\hline Ctenodiscus procurator & 28 & 28 & 195 & 373 & 0 & 0 & 0 & 0 & 0 & 0 & 593 & 34 & 45 & 1.296 \\
\hline Solaster regularis & 0 & 0 & 1 & 0 & 0 & 0 & 0 & 0 & 0 & 0 & 3 & 0 & 0 & 4 \\
\hline Ophiuroidea Indet 1. & 0 & 0 & 3 & 0 & 0 & 0 & 0 & 2 & 0 & 0 & 2 & 1 & 0 & 8 \\
\hline Ophiuroidea Indet 2. & 0 & 0 & 0 & 1 & 0 & 0 & 0 & 0 & 0 & 0 & 0 & 0 & 0 & 1 \\
\hline Ophiuroglypha lymani & 0 & 0 & 0 & 52 & 0 & 0 & 0 & 0 & 0 & 0 & 15 & 0 & 0 & 67 \\
\hline Ophiomyxa vivipara & 0 & 0 & 0 & 0 & 0 & 0 & 0 & 0 & 0 & 0 & 0 & 7 & 0 & 7 \\
\hline Holothuroidea Indet. & 4 & 0 & 1 & 2 & 0 & 5 & 1 & 0 & 0 & 0 & 1 & 0 & 0 & 14 \\
\hline Brisaster moseleyi & 0 & 0 & 88 & 0 & 0 & 0 & 0 & 0 & 0 & 0 & 0 & 1 & 29 & 118 \\
\hline
\end{tabular}




\begin{tabular}{|c|c|c|c|c|c|c|c|c|c|c|c|c|c|c|}
\hline Cont. Tabla 2 & $\begin{array}{c}\text { VGO } \\
36\end{array}$ & $\begin{array}{c}\text { VGO } \\
41\end{array}$ & $\begin{array}{c}\text { VGO } \\
57\end{array}$ & $\begin{array}{l}\text { VGO } \\
58 \mathrm{~A}\end{array}$ & $\underset{\mathrm{B}}{\mathrm{VGO}}$ & $\begin{array}{c}\text { VGO } \\
72\end{array}$ & $\underset{\mathrm{G}}{\mathrm{VGO}}$ & $\begin{array}{c}\text { VGO } \\
43\end{array}$ & $\begin{array}{c}\text { VGO } \\
52\end{array}$ & $\underset{\mathrm{P}}{\mathrm{VGO}}$ & $\begin{array}{c}\text { VGO } \\
58\end{array}$ & $\begin{array}{c}\text { VGO } \\
60\end{array}$ & $\begin{array}{c}\text { VGO } \\
49\end{array}$ & TOTAL \\
\hline Pseudechinus magellanicus & 0 & 0 & 0 & 2 & 0 & 0 & 0 & 1 & 0 & 0 & 0 & 9 & 0 & 12 \\
\hline Arbacia dufresnei & 0 & 0 & 0 & 0 & 0 & 0 & 0 & 0 & 0 & 0 & 0 & 1 & 0 & 1 \\
\hline Abatus sp. & 6 & 9 & 0 & 199 & 0 & 1 & 0 & 0 & 0 & 0 & 0 & 0 & 0 & 215 \\
\hline Etmopterus granulosus & 1 & 0 & 0 & 0 & 0 & 0 & 0 & 0 & 0 & 0 & 0 & 0 & 0 & 1 \\
\hline Batoidea Indet. & 0 & 0 & 1 & 0 & 0 & 0 & 0 & 0 & 0 & 0 & 0 & 0 & 0 & 1 \\
\hline Squalomorphi Indet. & 0 & 0 & 0 & 0 & 0 & 0 & 0 & 0 & 0 & 0 & 1 & 0 & 0 & 1 \\
\hline Macruronus magellanicus & 0 & 0 & 6 & 5 & 0 & 0 & 0 & 0 & 0 & 0 & 1 & 0 & 0 & 12 \\
\hline Ophidiidae Indet. & 0 & 0 & 0 & 0 & 0 & 0 & 0 & 0 & 0 & 0 & 2 & 0 & 0 & 2 \\
\hline
\end{tabular}

riqueza de especies y la profundidad se determinó el patrón de distribución de las estaciones de muestreo. Para ello, se utilizó un análisis de componentes principales (ACP). Previo al ACP, las variables fueron normalizadas según sugiere Clarke \& Gorley (2006). El ACP fue realizado utilizando el programa computacional PRIMER v6 ${ }^{\circledR}$ (Clarke \& Warwick, 1994).

\section{RESULTADOS}

Composición de especies, diversidad y dominancia numérica

En total, se recolectaron 3.124 individuos pertenecientes a 61 especies. Estos fueron clasificados en los phyla Mollusca (14 taxa de Bivalvia y 3 de Scaphopoda), Echinodermata (7 Asteroidea, 4 Ophiuroidea, 4 Echinoidea y 1 Holothuroidea), Annelida (12 taxa de Polychaeta), Arthropoda (8 Crustacea), Chordata (3 Pisces). Los phyla Brachiopoda, Cnidaria y Porifera estuvieron representados por un taxa cada uno (Tabla 2).

En término numéricos, el phylum Echinodermata fue dominante acumulando el $54 \%$ del total de individuos recolectados. La clase Asteroidea acumuló el $43 \%$, representada casi exclusivamente por Ctenodiscus procurator (41,5\%). La segunda clase en orden de importancia fue Echinoidea (11\%), dentro de la cual Abatus sp. representó un $6,9 \%$ del total recolectado.

Los moluscos bivalvos representaron el 24\% del total, con dos especies no identificadas: Carditidae Indet. (10,8\%) y Nuculoidea Indet. (8,2\%). Los moluscos escafópodos (15\%), estuvieron representados por Dentalium (F.) majorinum.

Las restantes 7 taxas tuvieron una representación igual o menor a $1 \%$ del total de individuos capturados (Fig. 2).

En término de número de individuos por estación de muestreo, en las estaciones asociadas al canal de las Montañas (VGO-58, VGO-58A y VGO-57) se recolectó el $85 \%$ del total de especímenes capturados. Estas mismas estaciones fueron las que presentaron la mayor riqueza de especies del área de estudio (Tabla 3).

La curva basada en el estimador CHAO1 no alcanza la asíntota y estima un máximo de 79 especies para el área de estudio (Fig. 3).

Estructura comunitaria y su relación con la profundidad

El ACP muestra que los dos primero componentes (CP1 y CP2) acumulan el 93\% de la varianza. El tamaño de los vectores refleja la importancia de la contribución de la variable dentro de los ejes de CP1 y CP2 (Fig. 4). En el eje del $\mathrm{CP} 1$, los resultados muestran una contribución casi igualitaria entre el número de individuos y el número de especies pero no para la profundidad. Una situación inversa se observa sobre el CP2, en donde la profundidad es la que contribuye mayoritariamente a la varianza (Tabla 3 ).

Basado en el ACP, se observa que a lo largo del eje de CP1 (de izquierda a derecha) existe clara tendencia desde estaciones con bajo valores de número de individuos y especies (e.g. VGO-52) a estaciones con alto número de individuos y especies (e.g. VGO-58). Mientras que a lo largo del eje del $\mathrm{CP} 2$ se distingue una clara tendencia batimétrica, desde estaciones de profundidad somera (VGO-B) a la de más alta profundidad (VGO-49).

Siguiendo el círculo de correlación (Fig. 4), se puede discriminar dos grupos de estaciones. Uno constituido por siete estaciones (VGO-49, VGO-41, VGO-72, VGO-G, VGO-36, VGO-43 y VGO-P), las cuales se caracterizan por presentar un promedio de profundidad de $334 \mathrm{~m}, 6,6$ especies y 51,7 individuos por estación. El segundo grupo, constituido por tres estaciones (VGO-58A, VGO- 
57 y VGO-60), se caracterizó por presentar un promedio menor de profundidad (191 m) pero un mayor número de especies e individuos (21,6 y 470,1 , respectivamente). Tres estaciones quedaron fuera, con un pobre nivel de correlación con las variables combinadas. Las estaciones VGO-52 y VGO-B con el rango de profundidad muy somero (<30 m.) y los menores números de individuos y la estación VGO-58 $(170 \mathrm{~m})$ con el número de individuos más alto encontrado en el muestreo.

\section{DISCUSIÓN}

Debido a su intrincada geografía y las características climáticas extremas, la región subantártica de Magallanes es una de las menos estudiadas dentro del sistema de fiordos y canales chilenos. No obstante, a partir de la organización de los cruceros CIMAR FIORDOS en 1996, este panorama ha ido cambiando paulatinamente. En este contexto, los resultados de este trabajo complementan de manera importante el nivel de conocimiento sobre la fauna bentónica que previamente se había descrito para las áreas frente a los Campos de hielo Sur (Ríos et al., 2005; Mutschke 2008) y, mas al sur, en el Estrecho de Magallanes (Ríos et al., 2005) utilizando el mismo método de muestreo.
Composición taxonómica y riqueza de especies

En términos de número de especies por lance, los resultados encontrados en el presente estudio son relativamente superiores a los descritos para los fiordos y canales frente a los Campos de Hielos Sur (Mutschke \& Gorny, 1999; Montiel et al., 2004; Ríos et al., 2005), pero menores a los registrados en el estrecho de Magallanes (Ríos et al., 2005). No obstante, en términos de número de individuos obtenidos con una red AGT, el presente estudio registró el valor más alto para la región de Magallanes (Tabla 4). Dentro del área estudiada, el sector del canal de las Montañas constituye el sitio de mayor interés en función de la abundancia y riqueza de especies.

Considerando a las áreas del estrecho de Magallanes y canales frente a los Campos de Hielo Sur, Echinodermata fue el taxón más diverso (47 especies), seguido por Polychaeta (46) y Mollusca (25). En el caso de Echinodermata, se identificaron 25 especies de Asteroidea mientras que en este estudio se encontraron siete especies, todas incluidas en el trabajo previo. Ctenodiscus procurator aparece también como una de las especies numéricamente más abundante (Ríos et al. 2005). En el caso de Polychaeta, la diferencia en el número de especies resultó ser más evidente. Respecto de Mollusca, en el muestreo del presente

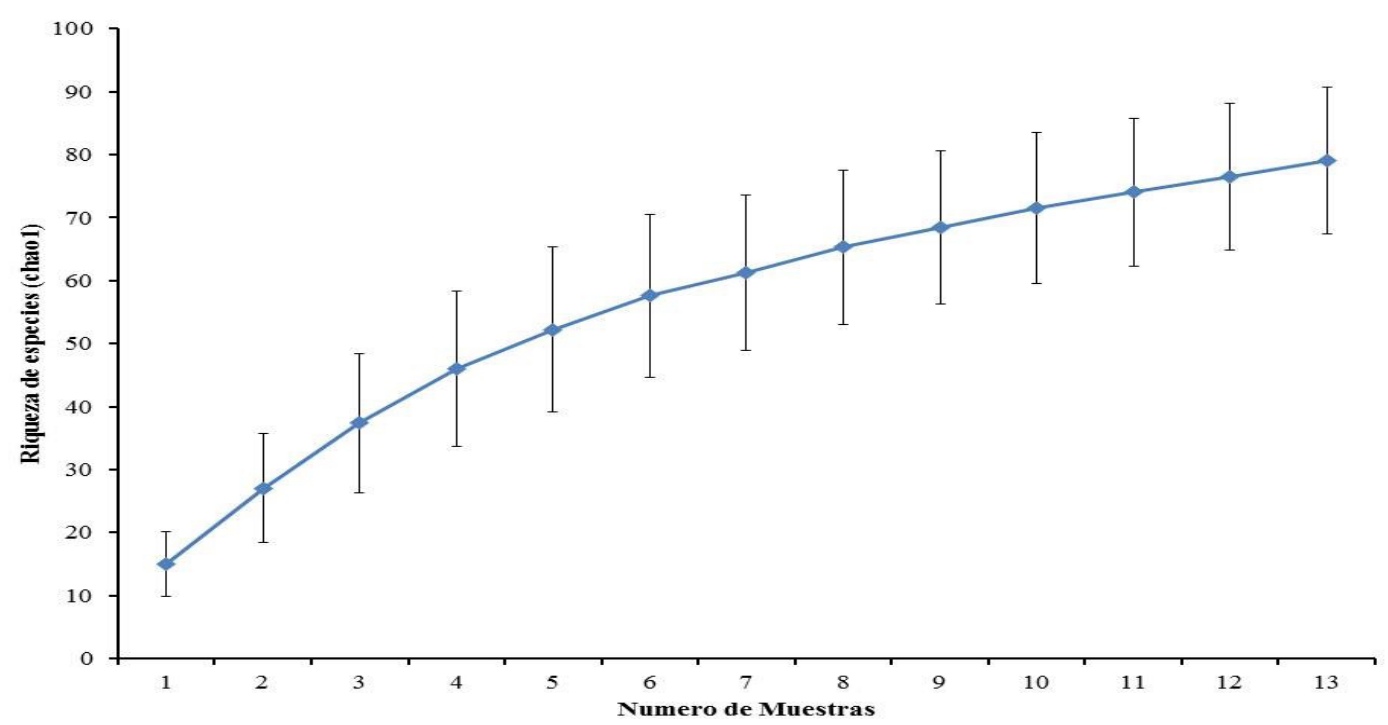

Fig. 3. Curva de riqueza de especies acumulada para los taxa bentónicos para 13 muestras usando el estimador de diversidad CHAO1 $(\mathrm{n}=3.124)$. Líneas representan la desviación estándar. 
Tabla 3. Resultados del análisis de componente principales (ACP) basado en los antecedentes obtenidos de las estaciones de muestreo de macrofauna epibentónica durante el crucero CIMAR 15 FIORDOS, utilizando una red de arrastre Agassiz modificada.

\begin{tabular}{lcc}
\hline Ejes ACP & CP 1 & CP 2 \\
\hline Valores propios & 1,8 & 0,995 \\
Proporcion de variaza & 59,8 & 33,2 \\
Variaza acumulada & 59,8 & 93 \\
Vectores propios & & \\
N. Especies & $-0,701$ & 0,121 \\
N. Individuos & $-0,706$ & 0,024 \\
Profundidad & 0,102 & 0,992 \\
\hline
\end{tabular}

estudio no se recolectaron especímenes de Gastropoda y la clase Bivalvia presentó un número relativamente similar de especies, aunque con al menos 6 especies no reportadas previamente.

\section{Gradiente batimétrico}

Los resultados del presente estudio mostraron una clara tendencia a formar dos ensambles de estaciones asociados con una gradiente de profundidad. El ensamble asociado a una profundidad relativamente somera (191 $\mathrm{m}$ en promedio) presentó los valores más altos en términos de número de individuos y riqueza de especie, en comparación con el ensamble de estaciones asociadas a mayores profundidades (334 $\mathrm{m}$ en promedio). Cabe destacar que dos estaciones (VGO-52 y VGO-B) con una profundidad menor a 30 metros presentaron los valores de riqueza de especies y numero de individuos más bajos del área de estudio. Similares patrones se han descrito para las comunidades bentónicas del estrecho de Magallanes y canal Beagle. En estos casos, se registraron dos comunidades someras y dos profundas, las cuales revelaron diferencias considerables en lo que se refiere a abundancia, número de taxones, diversidad y composición específica (Gutt et al. 1999). Otros patrones batimétricos han sido determinados para el taxon Polychaeta, distribuido en aguas someras y profundas del Paso Ancho, estrecho de Magallanes (Montiel et al. 2011). Este patrón también es común en las comunidades de áreas subantárticas, en donde las comunidades de aguas poco profundas son relativamente pobres debido a la influencia del hielo, con un aumento en la riqueza de especies hacia aguas más profundas, en donde el impacto de los disturbios generados por el hielo es menor (cf. Arntz et al. 2005).

\section{Consideraciones metodológicas}

Sin embargo, debido las limitaciones de la red de arrastre Agassiz, es necesaria tener ciertas precauciones con la interpretación de los patrones ecológicos resultantes. El estudio de las comunidades por macrozoobentónica por medio de las redes de arrastre entrega sólo una visión preliminar de la distribución de las comunidades epibénticos (Ríos et al. 2005), debido al tamaño de apertura de malla $(10 \mathrm{~mm}$ ) que solo obtiene los organismos de gran biomasa y la fracción más epibentónica de la comunidad fondo marino (Montiel et al., 2011). Al compararlo con otros estudios cualitativos en la región se observa una gran variación en la cantidad de organismos capturados (Tabla 4). Por lo que el efecto del tipo de muestreador (e.g. red de arrastre Rauschert) para sub o sobre estimar las abundancias y riquezas de especies se ha analizado en otros trabajos realizados en los sistemas marinos sublitorales de la región austral (e.g. Diez et al. 2009). Consecuentemente, para futuros estudios es altamente deseable una mayor densidad de estaciones de muestreo y la inclusión de métodos cuantitativos de muestreo complementarios a los cualitativos con una AGT.

En general, los resultados de este estudio concuerdan con los obtenidos en investigaciones previas (e.g. Ríos et al. 2005) y sugieren que la relativamente alta biodiversidad específica en los sectores de canales y fiordos australes se mantiene por un alto reemplazo de especies entre sitios, con sectores bien definidos y además diferenciados respecto de su composición biótica. Diferencias subregionales en regímenes de disturbios físicos (e.g. tasas de sedimentación, efectos de hoyas hidrográficas) como las presentes en el área de estudio, pueden ser las causas basales de la heterogeneidad bentónica que se ha observado en los sistemas marinos de Magallanes. Estos aspectos han sido sugeridos también para otros sistemas marinos que presentan una alta 


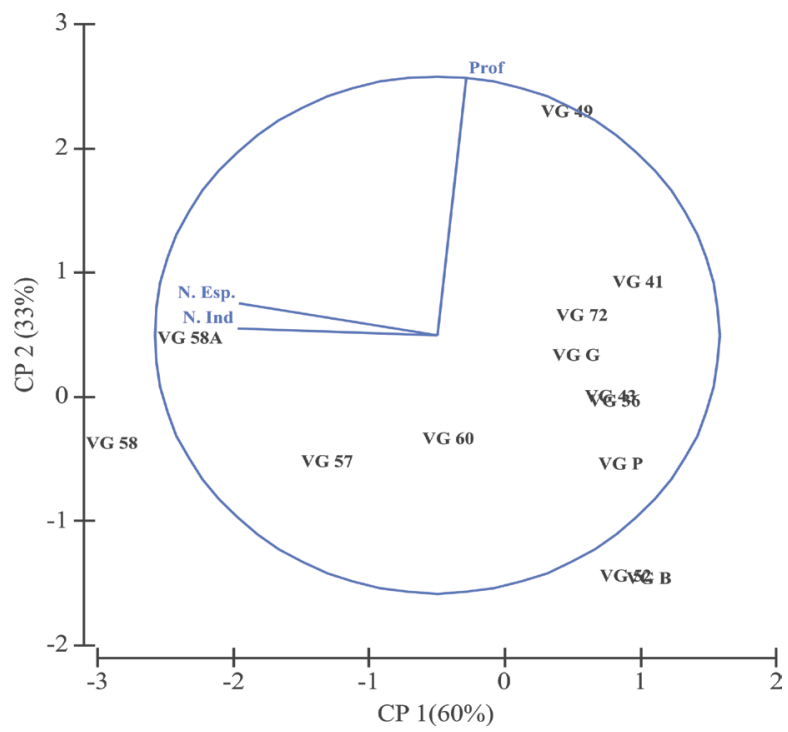

Fig. 4. Análisis de componentes principales para relacionar la estructura comunitaria de los organismos bentónicos con la profundidad. Datos obtenidos durante el Crucero CIMAR 15 FIORDOS.

variabilidad ambiental y que derivan de una historia glaciológica relativamente similar a la del área de estudio considerada en el presente trabajo (e.g. McCormick-Ray et al. 2011).

\section{AGRADECIMIENTOS}

El presente estudio fue apoyado financieramente por el Comité Oceanográfico Nacional a través del proyecto "Biodiversidad y
Estructura de las Asociaciones Macrofaunísticas Sublitorales del Sistema de Fiordos y Canales Australes" (CONA-C15F-09-08). El crucero CIMAR 15 FIORDOS permitió dar continuidad al desarrollo del proyecto quinquenal del programa CIMAR, favoreciendo la obtención de información actualizada sobre las características ecológicas y oceanográficas de los fiordos chilenos. Agradecemos la colaboración y facilidades otorgadas durante la realización del Crucero por el Capitán y la tripulación del buque oceanográfico "AGOR Vidal

Tabla 4. Investigaciones bentónicas cualitativas realizadas en ecosistemas marinos de la región de Magallanes. Rastra Agassiz (AGT), red de arrastre Rauschert (RR). (+) Solo contempla Polychaeta; (") Solo taxa superiores.

\begin{tabular}{|lccccl}
\hline Localidad & $\begin{array}{l}\text { Método } \\
\text { Captura }\end{array}$ & $\mathrm{N}^{\circ}$ Muestras & $\mathrm{N}^{\circ}$ individuos & $\mathrm{N}^{\circ}$ especies & \multicolumn{1}{c}{ Fuente } \\
\hline Fiordos y canales australes & AGT & 13 & 3.124 & 61 & $\begin{array}{l}\text { Presente estudio } \\
\text { Futschke \& Gorny, 1999; } \\
\text { Frente a Campos de Hielo Sur }\end{array}$ \\
& AGT & 20 & 1.019 & 82 & $\begin{array}{l}\text { Montiel et al. 2004; } \\
\text { Ríos et al. 2005 }\end{array}$ \\
Estrecho de Magallanes & AGT & 15 & 1.060 & 79 & Ríos et al. 2005 \\
Canal Beagle + & AGT & 23 & 178 & 23 & Montiel et al. 2007 \\
Canal Beagle * & RR & 45 & 32.686 & 30 & Diez et al. 2009 \\
\hline
\end{tabular}


Gormaz" de la Armada de Chile. Los autores expresan un especial reconocimiento al Sr. Jorge Ramírez Veloso y al Biólogo Marino Sr. Francisco Pizarro (Instituto de la Patagonia, Universidad de Magallanes) por su dedicado y valioso trabajo tanto a bordo de la embarcación como en los laboratorios del Instituto de la Patagonia. El Dr. Jim McAdam (Queen's University of Belfast) gentilmente tradujo el Resumen.

\section{LITERATURA CITADA}

Arntz, W. \& C. Ríos (1999). Magellan-Antarctic: Ecosystem that drifted apart. Scientia Marina, 63 (suppl. 1): 518 pp

Arntz, W.E., S. Thatje, D. Gerdes, J-M. Gili, J. Gutt, U. Jacob, A. Montiel, C. Orejas \& N. Teixido (2005). The Antarctic-Magellan connection: macrobenthos ecology on the shelf and upper slope, a progress report. Scientia Marina, 69 (Suppl. 2): 237-269

Bernasconi, I. (1969). Equinodermos argentinos. Revista del Museo Argentino de Ciencias Naturales "Bernardino Rivadavia", 9(9): 197-210

Bernasconi, I. \& M.M. D’Agostino (1971). Ofiuroideos argentinos. Physis, 30(81): 447-469

Bernasconi, I \& M.M. D'Agostino (1977). Ofiuroideos del mar epicontinental argentino. Revista del Museo Argentino de Ciencias Naturales "Bernardino Rivadavia", 5(1): 66-114

Brattström, H. \& A. Johanssen (1983). Ecological and regional zoogeography of the marine benthic fauna of Chile. Sarsia, 68: 289-339

Camus, P.A. 2001. Biogeografía marina de Chile continental. Revista Chilena de Historia Natural, 74: 587-617

Cárdenas, J., C. Aldea \& C. Valdovinos (2008). Chilean Marine Mollusca of Northern Patagonia Collected during the Cimar-10 Fjords Cruise. Gayana, 72(2): 202-240

Castellanos, Z.A. de. (1988). Catálogo descriptivo de la malacofauna marina magallánica 1. Comité de Investigaciones Científicas. Buenos. Aires, Argentina 1: 1-41

Castellanos, Z.A. de. (1992). Catálogo descriptivo de la malacofauna magallánica 8.
Neogastropoda, Columbellidae, Pyrenidae, Cominellidae y Fasciolariidae. Comisión de Investigaciones Científicas, Provincia de Buenos Aires, Argentina, $41 \mathrm{pp}$

Castellanos, Z.A. de. (1994). Los Invertebrados. Primera Parte. Moluscos. Tomo 3: 1-206

Clapperton, C.M., D.E. Sugden, D.S. Kaufman \& R.D. McCulloch (1995). The last Glaciation in Central Magellan Strait, Southernmost Chile. Quaternary Research, 44: 133-148

Clarke, K.R. \& R.M. Warwick. 1994. Changes in marine communities: an approach to statistical analysis and interpretation. Plymouth: Plymouth Marine Laboratory.

Clarke, K. R. \& R. N. Gorley (2006). PRIMER v6: User Manual Tutorial. Primer - E: Plymouth, UK. $190 \mathrm{pp}$

Colwell, R.K. (2006). EstimateS 8.2 User's Guide. /http://viceroy.eeb.uconn.edu/ EstimateSPages/EstSUsersGuide/S.

Costello, M.J., M. Coll, R. Danovaro, P. Halpin, H. Ojaveer \& P. Miloslavich (2010). A census of marine biodiversity knowledge, resources, and futures challenges. PLoS ONE, 5(8): $1-15$

Diez, M.J., M.C. Romero, S. Obenat, M.J. Albano \& F. Tapella (2009). Distribution of benthic invertebrates in the Beagle Channel, Argentina. Anales Instituto Patagonia (Chile), 37: 29-40

Escribano, R., M. Fernández \& A. Aranís (2003). Physical-chemical processes and patterns of diversity of the Chilean eastern boundary pelagic and benthic marine ecosystems: and overview. Gayana, 67(2): 190-205

Fernández, M., E. Jaramillo, P. Marquet, C. Moreno, S. Navarrete, P. Ojeda, C. Valdovinos \& J. Vásquez (2000). Diversity, dynamics and biogeography of Chilean benthic nearshore ecosystem: and overview and guideline for conservation. Revista Chilena de Historia Natural, 73:629-662

Forcelli, D. (2000). Moluscos magallánicos. Guía de moluscos de Patagonia y sur de Chile. Vásquez Mazzini Editores, Buenos Aires, Argentina.

Gray, J.S. (2001). Antarctic marine benthic diversity in a world-wide context. Polar Biology, 24: 633-641 
Gutt, J., E. Helsen, W. Arntz \& A. Buschmann (1999). Biodiversity and community structure of the mega-epibenthos in the Magellan region (South America). Scientia Marina, 63 (Suppl. 1): 155-170

Hartmann-Schröder, G. (1965). Zur Kenntnis des Sublitorals der chilenischen Küste unter besonderer Berücksichtigung der Polychaeten und Ostracoden. T1.II. Die Polychaeten des Sublitorals. Mitteilungen Aus Dem Hamburgischen Zoologischen Museum Und Institut, 62 (suppl.): 59-305

Kilian, R., O. Baeza, T. Steinke, M. Arévalo, C. Ríos \& C. Schneider (2007). Late Pleistocene to Holocene marine transgression and thermohaline control on sediment transport in the western Magellanes fjord system of Chile (53ㅇ). Quaternary International, 161: 90-107

Lancellotti, D.A. \& J. Vásquez (2000). Zoogeografía de macroinvertebrados bentónicos de la costa de Chile: contribución para la conservación marina. Revista Chilena de Historia Natural, 73: 99-129

Lamilla, J \& C. Bustamante. 2005. Guía para el reconocimiento de: tiburones, rayas y quimeras de Chile. Oceana, 17: 1-80

McCormick-Ray, J., R.M. Warwick \& G. Carleton Ray (2011). Benthic macrofaunal composition variations in the northern Bering Sea. Marine Biology, 158: 1365$1376 \mathrm{ck}$

McCulloch, R.D., C.M. Clapperton, J. Rabasa \& A. P. Currant (1997). The glacial and postglacial environmental history of FuegoPatagonia. In: McEwan, C., L. A. Borrero \& A. Prieto (eds.) Patagonia: Natural history, prehistory and ethnography at the uttermost end of the Earth. British Museum Press, London. pp 12-31

Menzies, R.J. (1962). The Zoogeography, ecology, and systematics of the chilean marine isopods. Reports of the Lund University Chile Expedition 1948-49.

Montiel, A., C. Ríos, E. Mutschke \& N. Rozbaczylo (2004). Poliquetos de fiordos y canales adyacentes al Campo Patagónico Sur, Chile (Annelida:Polychaeta) Ciencia y Tecnología del Mar, 27(1): 49-67
Montiel, A., D. Gerdes, B. Hilbig \& W.E. Arntz (2005). Polychaete assemblages on the Magellan and Weddell Sea shelves: comparative ecological evaluation. Marine Ecology Progress Series, 297: 189-202

Montiel, A. Quiroga, E. \& D. Gerdes (2011). Diversity and spatial distribution patterns of polychaete assemblages in the Paso Ancho, Straits of Magellan Chile. Continental Shelf Research, 31: 304-314

Mutschke, E. (2008). Macrobenthic biodiversity and community structure in austral Chilean channels and fjords. In: Silva, N. \& S. Palma. (eds.) Progress in the oceanographic knowledge of Chilean interior waters, from Puerto Montt to Cape Horn. Comité Oceanográfico Nacional - Pontificia Universidad Católica de Valparaíso. pp. 133 $-141$

Mutschke, E. \& M. Gorny (1999). The benthic decapod fauna in the channels and fjords along the South Patagonian Icefield, Southern Chile. In: W. Arntz \& C. Ríos (eds.) Magellan-Antarctic: Ecosystems that drifted apart. Scientia Marina 63 (Suppl. 1): 315-319

Osorio, C. \& D. Reid (2004). Moluscos marinos intermareales y submareales entre la Boca del Guafo y el Estero Elefantes, sur de Chile. Investigaciones Marinas, 32: 31-89

Retamal, M.A. (1974). Contribución al conocimiento de los crustáceos decápodos de la región Magallánica. Gayana (Zoología), 31: $1-23$

Ríos, C. (2007). Marine benthic communities of the Magellan Region, Southern Chile: contribution of different habitats to the overall biodiversity. $\mathrm{PhD}$ Thesis, Alfred Wegener Institut Für Polar und Meeresforschung-University of Bremen, Germany. 122 pp.

Ríos, C., E. Mutschke \& A. Montiel (2010a.) Estructura de la comunidad macrofaunística bentónica en la boca oriental del estrecho de Magallanes, Chile austral. Anales Instituto Patagonia (Chile), 38(1): 83-96

Ríos, C., E. Mutschke, A. Montiel \& F. Pizarro (2010b.) Biodiversidad y estructura de las asociaciones macrofaunísticas sublitorales 
del sistema de fiordos y canales australes. Crucero CIMAR 15 FIORDOS. Informes Preliminares (Oct. 2010). Comité Oceanográfico Nacional. pp. 149-167

Ríos, C., W. Arntz, D. Gerdes, E. Mutschke \& A. Montiel (2007). Spatial variability of the benthic assemblages associated to the holdfasts of the kelp Macrocystis pyrifera in the Straits of Magellan, Chile. Polar Biology, 31: 89-100

Ríos, C., E. Mutschke, A. Montiel, D. Gerdes \& W. Arntz (2005). Soft-bottom macrobenthic faunal associations in the southern Chilean glacial fjord complex. Scientia Marina, 69 (Suppl. 2): 225-236

Ríos, C. E. Mutschke \& E. Morrison (2003). Biodiversidad bentónica sublitoral en el estrecho de Magallanes, Chile. Revista de Biología Marina y Oceanografía, 38: 1-12

Rozbaczylo, N. (1980). Clave para el reconocimiento de familias de Anélidos poliquetos del mar chileno. Studies on Neotropical Fauna and Environment, 15:167-196

Silva, N. (2010). Distribución de temperatura, salinidad, oxígeno disuelto y nutrientes, en la sección canal Smyth a canal Señoret. Crucero CIMAR 15 FIORDOS. Informes Preliminares (Oct. 2010). Comité Oceanográfico Nacional. pp. 31-41

Silva, N. \& S. Palma (2008). The CIMAR Program in the austral Chilean channels and fjords. In: Silva, N. \& S. Palma. (eds.) Progress in the oceanographic knowledge of Chilean interior waters, from Puerto Montt to Cape Horn. Comité Oceanográfico Nacional - Pontificia Universidad Católica de Valparaíso. pp. 11-15

Sievers, H.A. \& N. Silva (2006). Masas de agua y circulación en los canales y fiordos australes. En: Silva, N \& S. Palma (eds.) Avances en el conocimiento oceanográfico der las aguas interiores chilenas, Puerto Montt a cabo de Hornos. Comité Oceanográfico Nacional-Pontificia Universidad Católica de Valparaiso. pp. 53-58

Valdovinos, C., S. Navarrete \& P. Marquet (2003). Mollusk species diversity in the southern Pacific: why are there more species towards the pole? Ecography, 26: 139-144 